\title{
Canine oral cavity T-cell lymphoma - histopathological and immunohistochemical study
}

\author{
KATARZYNA PAŹDZIOR-CZAPULA, IWONA OTROCKA-DOMAGAŁA, \\ MICHAŁ GESEK, MATEUSZ MIKIEWICZ
}

\author{
Department of Pathological Anatomy, Faculty of Veterinary Medicine, \\ University of Warmia and Mazury in Olsztyn, Oczapowskiego 13, 10-719 Olsztyn, Poland
}

Paździor-Czapula K., Otrocka-Domagała I., Gesek M., Mikiewicz M.

\section{Canine oral cavity T-cell lymphoma - histopathological and immunohistochemical study}

Summary

This study presents a case of an unusually located canine T-cell lymphoma. A 5-year-old female dachshund was presented with a tumour located in the buccal mucosa. The tumour was excised, fixed, processed routinely for histopathology and stained. Microscopically, a dense infiltration of round cells with scant cytoplasm, large nuclei and numerous mitotic figures was detected within the mucosa. The tumour was diagnosed as a roundcell tumour. Subsequently, additional tumours developed in the mandibular and hock joint areas. The primary tumour was stained immunohistochemically using an antibody panel (CD3, MHCII, mast cell tryptase, CD18, CD79a). The tumour cells showed variable cytoplasmic expression of CD3, moderate-to-strong cytoplasmic or membranous expression of MHCII, and they were mast cell tryptase, CD18 and CD79a negative. The final diagnosis was T-cell lymphoma. The dog passed away within the next two months. This study revealed, that immunohistochemistry is necessary to diagnose canine oral cavity round cell tumours.

Keywords: dog, immunohistochemistry, round-cell tumour, oral lymphoma

Canine lymphomas occur most often as multicentric diseases, causing generalized enlargement of the peripheral lymph nodes, but can also be localized in the skin, mucous membranes or any other organ (4). The mucous membranes can either be the primary site of some types of lymphomas (i.e. marginal zone B-cell lymphoma of mucosa-associated lymphoid tissue (MALT), T-cell intestinal lymphoma), or can be secondarily involved in the generalized disease (6). However, oral masses are uncommon in lymphoma (2). Due to an unusual location, oral lymphomas in dogs must be differentiated from other round-cell tumours of the oral cavity. The antibody panel designed primarily for the differentiation of canine cutaneous round-cell tumours includes major histocompatibility complex class II (MHCII) and CD18, which are markers of histiocytic cells; tryptase, which is specific for mast cells; and the lymphocytic markers CD3 and CD79a (1). The same panel can also be used for the differentiation of the main types of oral cavity round-cell tumours. This study presents an unusual case of canine T-cell lymphoma, located in the buccal mucosa.

\section{Case description}

A 5-year-old female dachshund was presented to the referring veterinarian with a tumour measuring approximately $3 \mathrm{~cm}$ located in the buccal mucosa of the oral cavity. The tumour was excised surgically, immediately fixed in $10 \%$ buffered formalin, embedded in paraffin and cut. The sections were stained with Mayer's haematoxylin and eosin (HE) and May-Grünwald-Giemsa stain (MGG; staining kit, Bio-Optica, Milan, Italy). Microscopically, a dense infiltration of round to polygonal cells with scant cytoplasm lacking metachromatic granules and moderate anisocytosis and anisokaryosis, extending from the superficial to the deep mucosa, was observed. The nuclei were large, round to oval, with coarse chromatin and numerous nucleoli. The mean number of mitotic figures, counted in 10 adjacent high power fields (HPFs, $400 \times$ ), was 11/HPF (Fig. 1). The superficial epithelium was ulcerated. The tumour cells were accompanied by single mast cells, as shown by the MGG stain. At the tumour periphery, small to moderate subepithelial and perivascular infiltrations of plasma cells were detected. Additionally, some melanin-laden macrophages were observed under the superficial epithelium. The morphological diagnosis was a poorly differentiated round- 
cell tumour. Two months later, additional tumours developed in the submandibular and hock joint areas. The primary tumour was sectioned, mounted on silanized glass slides and stained immunohistochemically using an antibody panel (CD3, MHCII, mast cell tryptase, CD18, CD79a) and a visualization system based on an immunoperoxidase method, with 3.3-diaminobenzidine (DAB) as a substrate (Tab. 1). The specimens were counterstained with Mayer's haematoxy-

lin. For the negative control, the primary antibody was either replaced by mouse IgG1 (Dako, Glostrup, Denmark) in an appropriate dilution (MHCII, mast cell tryptase, CD18, CD79a) or omitted (CD3). For the positive control, normal canine tonsil (CD3, MHCII, CD79a), well-differentiated canine cutaneous mast cell tumour (mast cell tryptase), and canine pyogranuloma (CD18) sections were processed together with the evaluated slides. The whole immunohistochemical procedure was conducted twice, with similar results. Approximately $60 \%$ of the tumour cells showed variable (weak, moderate or strong) cytoplasmic expression of CD3 (Fig. 2). Some of the strongly CD3-immunoreactive cells were observed intravascularly. Approximately $80 \%$ of the tumour cells showed moderate-to-strong cytoplasmic or membranous expression of MHCII (Fig. 3). Strong membranous expression of MHCII was also detected in bystander cells at the tumour periphery. The tumour cells were mast cell tryptase, CD18 and CD79a negative. Strong cytoplasmic expression of mast cell tryptase was observed in a few cells scattered within the tumour stroma, presumably in normal mast cells. CD79a immunoreactive plasma cells were scattered within the tumour stroma and formed

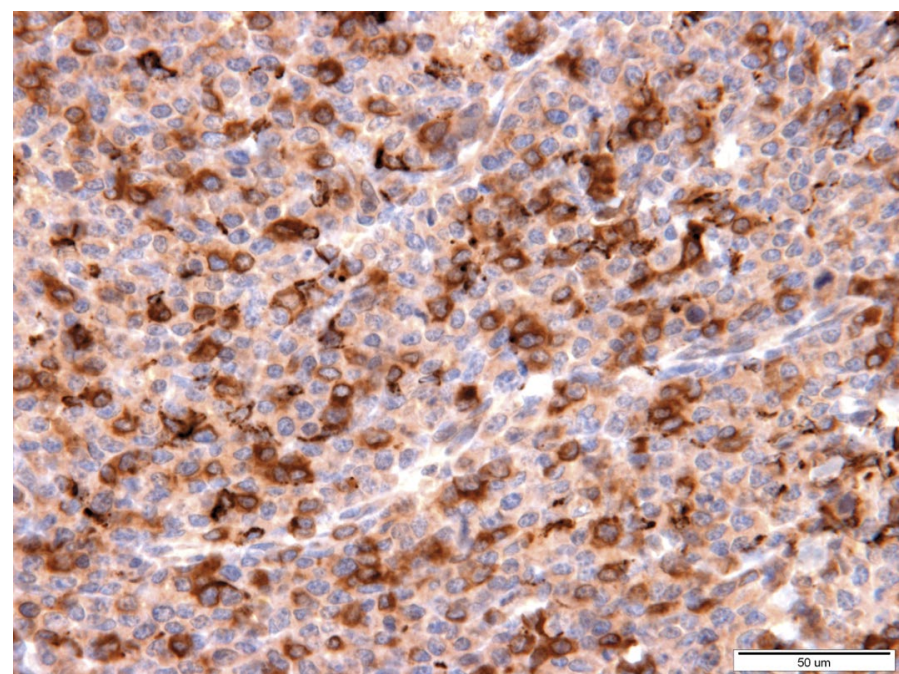

Fig. 2. The majority of tumour cells showed mild, moderate or strong cytoplasmic expression of CD3. IHC

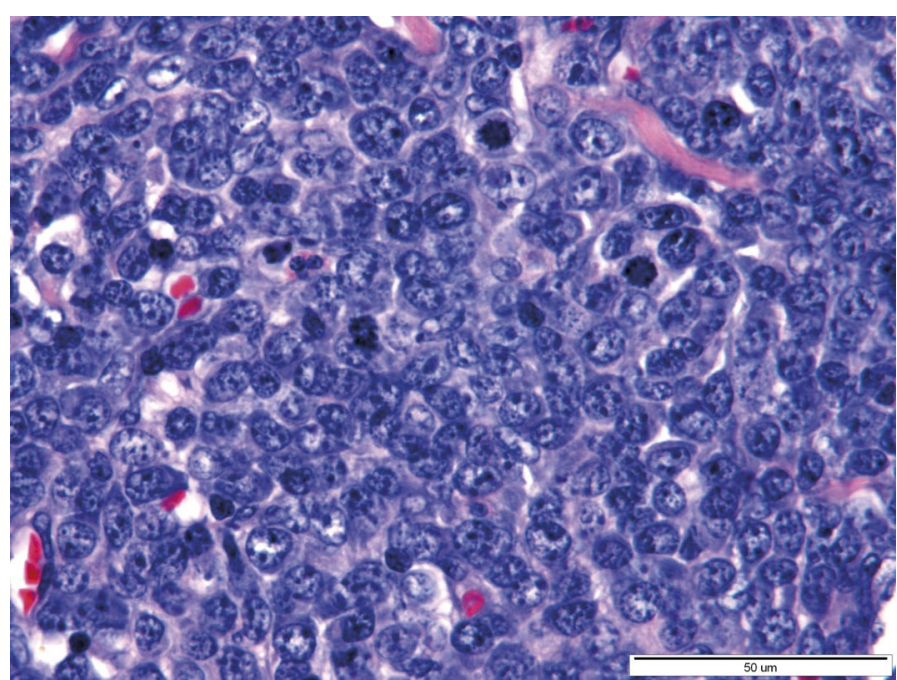

Fig. 1. A dense infiltration of round-to-polygonal cells with scant cytoplasm and large, round-to-oval nuclei with coarse chromatin. Some of the tumour cells undergo mitosis. The tumour cells are accompanied by single eosinophils. HE

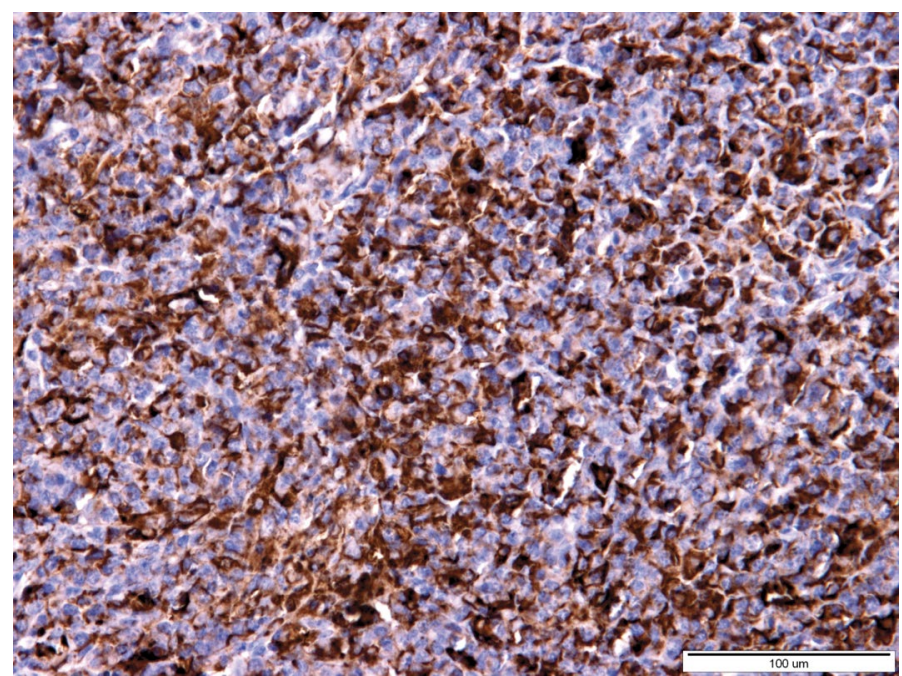

Fig. 3. The tumour cells showed moderate-to-strong cytoplasmic or membranous expression of MHCII. IHC 
perivascular or band-like infiltrates under the superficial epithelium. Based on the immunohistochemistry results, T-cell lymphoma was diagnosed. The dog passed away within the next 2 months. Necropsy was not performed.

\section{Discussion}

The T-cell lymphoma described in this study can be classified as an extranodal, peripheral T-cell lymphoma, not otherwise specified (PTCL-NOS), according to the WHO classification (6). Although it was first presented as a solitary tumour of the buccal mucosa, the mitotic count of the evaluated tumour suggested a high grade of malignancy (4). The biological behaviour of the tumour was presumably highly aggressive, but unfortunately, neither a histopathological examination of the subsequently formed tumours nor a necropsy was performed.

The presented tumour was first histologically diagnosed as a round-cell tumour. The differential diagnoses of canine round-cell tumours located in the oral cavity include lymphoma, transmissible venereal tumour, melanoma, neuroendocrine tumour, plasma cell tumour and mast cell tumour (5). Furthermore, poorly differentiated sarcomas, particularly alveolar rhabdomyosarcoma, should also be considered (3). The diagnosis of the presented tumour was based on the CD3 immunoexpression in the tumour cells. Additionally, the tumour cells were MHCII-positive and CD18-negative. Most canine lymphomas express either CD3 or CD79a, but there is also a subset of lymphomas that are CD3- and CD79a-negative. These lymphomas are referred to as null-cells (4). Although canine T and B lymphocytes express MHCII and CD18, the expression of these markers in canine lymphomas is variable (1).

In conclusion, the present study described an unusually located T-cell lymphoma in a dog. An extended diagnostic panel including a wide range of antibodies should be always implemented in the diagnosis of the oral cavity round-cell tumours in dogs.

\section{References}

1. Fernandez N. J., West K. H., Jackson M. L., Kidney B. A.: Immunohistochemical and histochemical stains for differentiating canine cutaneous round cell tumors. Vet. Pathol. 2005, 42, 437-445.

2. Ito T., Hisasue M., Neo S., Ishikawa T., Shida T., Watanabe T., Enomoto Y., Okuda M., Tsuchiya R., Madarame H., Ogata M., Yamada T.: A case of atypical canine lymphoma with oral mass and multiple osteolysis. J. Vet. Med. Sci. 2007, 69, 977-980

3. Otrocka-Domagała I., Paździor-Czapula K., Gesek M., Koda M., Mikiewicz M., Mikolajczyk A.: Aggressive, solid variant of alveolar rhabdomyosarcoma with cutaneous involvement in a juvenile labrador retriever. J. Comp. Pathol. 2015, 152, 177-181.

4. Ponce F., Marchal T., Magnol J. P., Turinelli V., Ledieu D., Bonnefont C., Pastor M., Delignette M. L., Fournel-Fleury C.: A morphological study of 608 cases of canine malignant lymphoma in France with focus on comparative similarities between canine and human lymphoma morphology. Vet. Pathol. 2010, 47, 414-433.

5. Taci Cangul I.: Improved classification, diagnosis and prognosis of canine round cell tumours. Vet. Sci. Tomorrow 2001, 4, 1-19.

6. Valli V. E., Jacobs R. M., Parodi A. L., Venau W., Moore P. F.: Histological Classification of Hematopoietic Tumors of Domestic Animals. World Health Organization International Histological Classification of Tumors of Domestic Animals, Volume VIII, $2^{\text {nd }}$ Edit., Armed Forces Institute of Pathology, American Registry of Pathology, The World Health Organization Collaborating Center for Worldwide Reference on Comparative Oncology, Washington, D.C. 2002.

Corresponding author: Katarzyna Paździor-Czapula, DVM, PhD, Department of Pathological Anatomy, Faculty of Veterinary Medicine, University of Warmia and Mazury in Olsztyn, Oczapowskiego 13, 10-719 Olsztyn, Poland; e-mail: katarzyna.pazdzior@uwm.edu.pl 